PROCEEDINGS OF THE

AMERICAN MATHEMATICAL SOCIETY

Volume 129, Number 9, Pages 2771-2775

S 0002-9939(01)05784-7

Article electronically published on April 16, 2001

\title{
A NORMAL COUNTABLY COMPACT NOT ABSOLUTELY COUNTABLY COMPACT SPACE
}

\author{
OLEG PAVLOV
}

(Communicated by Alan Dow)

\begin{abstract}
We construct an example of a normal countably compact not absolutely countably compact space. We also prove that every hereditarily normal countably compact space is absolutely countably compact and suggest a method for construction of hereditarily normal spaces without property $(a)$.
\end{abstract}

\section{INTRODUCTION}

M. V. Matveev [Mat1] called a space $X$ absolutely countably compact, or acc, provided for every open cover $\mathcal{U}$ of $X$ and every dense $F \subset X$ there is a finite $G \subset F$ such that $\operatorname{St}(G, \mathcal{U})=X$. (As usual, the star $\operatorname{St}(G, \mathcal{U})$ of a cover $\mathcal{U}$ with respect to a subset $G$ is $\cup\{U \in \mathcal{U}: U \cap G \neq \emptyset\}$.) This definition was motivated by a characterization of countably compact spaces in terms of stars of finite subsets (see [Fle]), so that every absolutely countably compact space is countably compact (we assume all spaces to be Hausdorff). It was established in Mat1 that all compact spaces are acc and that there are countably compact not acc spaces and acc not compact spaces. However, a part of the picture was missing.

Question 1. (Due to A. V. Arhangel'skii in [Mat1, repeated in [JMS], [RSV], and $\mathrm{Vau}$.) Is there a normal countably compact not absolutely countably compact space?

Motivation for this question lies not only in the fact that all countably compact not acc spaces considered in Mat1 and subsequent papers are clearly not normal but also the fact that many natural properties in conjunction with countable compactness imply absolute countable compactness (several such properties were listed in paper [RSV] which was devoted to understanding when a countably compact space is acc). Question 1 is also backed by the fact that in many cases normality alone implies a weaker form of acc, called property $(a)$.

This property was extracted by M. V. Matveev as exactly the property in presence of which countably compact spaces are acc. Namely, $X$ has property $(a)$ if for every open cover $\mathcal{U}$ and every dense $F \subset X$ there is a closed discrete $G \subset X$ such that $G \subset F$ and $\operatorname{St}(G, \mathcal{U})=X$. Even though the definition of property $(a)$ has

Received by the editors April 4, 1998 and, in revised form, November 9, 1999.

1991 Mathematics Subject Classification. Primary 54D30, 54G20.

Key words and phrases. Countably compact, absolutely countably compact, normal, hereditarily normal, property $(a)$. 
nothing to do with normality, it was observed in [Mat2, [Vau and subsequent papers that a strange connection between these properties does exist. Many theorems remain true if "normality" is mechanically replaced with "property $(a)$ ".

A list of normality-type properties which imply $(a)$ includes monotone normality and almost 2-fully normality RSV. In fact, a construction of a normal space without property $(a)$ requires special efforts. Such an example was constructed in [JMS], unfortunately under the assumption of the existence of a $Q$-set. Yet it turned out that an example of a normal countably compact not absolutely countably compact space does exist. The main result of our note is a construction of such an example in ZFC. We also show that any hereditarily normal countably compact space $i s$ acc (this result was proved earlier by W. Pack in $\mathrm{Pac}$ under the assumption of PFA). Finally, we expand the mentioned example from [JMS] (which appeared to be hereditarily normal) to produce more examples of (hereditarily) normal spaces without property $(a)$.

\section{Notation and the Definition of the example}

Our notation is standard and follows this of Eng]. Ordinals are thought of as collections of the smaller elements. For a space $X$ and an index set $I, X^{I}$ denotes the $I$-th power of $X$ equipped with the product topology. If $x \in X$, we write $x^{I}$ rather than $\{x\}^{I}$ to denote a point of $X^{I}$. For a family of spaces $\left\{X_{i}: i \in I\right\}$ and $a \in \prod\left\{X_{i}: i \in I\right\}$, a $\Sigma$-product (= $\Sigma_{\omega}$-product) of $\left\{X_{i}: i \in I\right\}$ which contains $a$ is the set of all $x \in \prod\left\{X_{i}: i \in I\right\}$ such that at most countably many coordinate of $x$ are different from the corresponding coordinates of $a . \Sigma_{\omega_{1}}$-products are defined similarly. Now let $K^{\prime}=\prod\left\{D_{\alpha}: \alpha \in \omega_{2}\right\}$, where each $D_{\alpha}$ is homeomorphic to $\{0,1\}$. For each $\alpha \in \omega_{2}$ let $X^{\prime}{ }_{\alpha} \subset K^{\prime}, X^{\prime}{ }_{\alpha}=\Sigma\left\{D_{\beta}: \beta \in \alpha\right\} \times 0^{\omega_{2} \backslash \alpha}$, where $1^{\alpha} \times 0^{\omega_{2} \backslash \alpha} \in$ $X_{\alpha}^{\prime}$; let also $X^{\prime}=\Sigma\left\{D_{\beta}: \beta \in \omega_{2}\right\} \ni 1^{\omega_{2}}$. We denote $K=K^{\prime} \times\left(\omega_{2}+1\right)$, where the second multiple is endowed with the natural topology of a linearly ordered space. Let $\omega_{2}^{\prime}=\left\{\alpha \in \omega_{2}: c f(\alpha) \leq \omega\right\}$ and let $X \subset K, X=\cup\left\{X_{\alpha}^{\prime} \times\{\alpha\}: \alpha \in \omega_{2}^{\prime}\right\}$. Let $\omega_{2}^{1}, \omega_{2}^{2}$ be disjoint subsets of $\omega_{2}$ of cardinality $\omega_{2}$ whose union equals $\omega_{2}$. Let $Y^{\prime} \subset K^{\prime}, Y^{\prime}=1^{\omega_{2}^{1}} \times \Sigma_{\omega_{1}}\left\{D_{\alpha}: \alpha \in \omega_{2}^{2}\right\}$, where $1^{\omega_{2}} \in Y^{\prime}$. Finally, $Y=Y^{\prime} \times\left\{\omega_{2}\right\}$ and $T=X \cup Y$. T has the topology of a subspace of $K$ and it is our example. This fact follows from the analysis performed in Section 3.

\section{The PRoOF}

We need to introduce some more notation. $\pi^{1}$ and $\pi^{2}$ denote projections of $K$ on the first and second coordinates respectively. For any $F \subset K^{\prime}$ and $\alpha \in \omega_{2}, \pi_{\alpha}(F)$ is the projection of $F$ on the $\alpha$-th coordinate, and $\pi_{<\alpha}(F)$ is a projection of $F$ on $\prod\left\{D_{\beta}: \beta \in \alpha\right\}$. For any $G \subset K$ and $\alpha \in \omega_{2}, G_{\alpha}$ denotes $G \cap\left(\pi^{2}\right)^{-1}(\{\alpha\})$ and $G_{<\alpha}$ denotes $G \cap\left(\pi^{2}\right)^{-1}(\alpha)$. In particular, for each $\alpha \in \omega_{2}, X_{\alpha}=X_{\alpha}^{\prime} \times\{\alpha\}$.

It is known that $\Sigma$-products and $\Sigma_{\omega_{1}}$-products of metrizable compacta are normal, countably compact and $C$-embedded in the product of these compacta. Therefore $Y$ and each $X_{\alpha}, \alpha \in \omega_{2}^{\prime}$, are normal, countably compact and $C$-embedded in $K$.

Our first task is to show that $X$ is normal, countably compact and $C$-embedded in $K$.

Claim 1. $X$ is normal, countably compact and $C$-embedded in $K$. 
Proof. Let $F \subset X,|F|=\omega$. If there is $\alpha \in \omega_{2}^{\prime}$ such that $\left|F_{\alpha}\right|=\omega$, then $F_{\alpha}$ has an accumulation point in $X_{\alpha}$ since $X_{\alpha}$ is countably compact. Otherwise $\left|\pi^{2}(F)\right|=\omega$ and we can assume that $\pi^{2}(F)$ is ordered of type $\omega$. Let $F=\left\{x_{n}: n \in \omega\right\}$, where $\pi^{2}\left(x_{i}\right)<\pi^{2}\left(x_{j}\right)$ for $i<j$. For any $x \in F$ let $\phi(x) \in X^{\prime}, \phi(x)$ has the same $\alpha$-th coordinate as $\pi^{1}(x)$ for each $\alpha<\pi^{2}(x)$ and all the other coordinates of $\phi(x)$ are 1. $\phi(F)$ contains a sequence which converges to some $x^{\prime} \in X^{\prime}$ since $\phi(F) \subset X^{\prime}$ and $X^{\prime}$ is Frechet. Then clearly the preimage of this sequence converges to point $\left(\pi_{<\beta}\left(x^{\prime}\right) \times 0^{\omega_{2} \backslash \beta}, \beta\right)$, where $\beta=\sup \left(\pi^{2}(F)\right)$. Therefore $X$ is countably compact.

Now assume that $X$ is not normal $C$-embedded in $K$. Then there are exist closed disjoint $F, G \subset X$ such that $\bar{F}^{K} \cap \bar{G}^{K} \neq \emptyset$. Let $\gamma \leq \omega_{2}$ be the smallest ordinal such that ${\overline{F_{\leq \gamma}}}^{K} \cap{\overline{G_{\leq \gamma}}}^{K} \neq \emptyset$ (where $F_{\leq \gamma}=F_{<\gamma+1}$ and $G_{\leq \gamma}=G_{<\gamma+1}$ ), and let $a \in K_{\gamma} \cap \bar{F}^{K} \cap \bar{G}^{K}$. Since $a \notin \overline{F \backslash F_{\leq \gamma}} K^{K} \cup \overline{G \backslash G_{\leq \gamma}} K$, we can assume that $F=F_{\leq \gamma}$ and that $G=G_{\leq \gamma}$. Fix $\delta<\gamma$. The sets $F_{\geq \delta}=\bar{F} \backslash F_{<\delta}$ and $G_{\geq \delta}=G \backslash G_{<\delta}$ contain $a$ in their closures because of the minimality of $\gamma$. For any $h \in X_{\geq \delta}$ and $H \subset X$, we denote $\pi_{<\delta}^{1}(h)=\pi_{<\delta}\left(\pi^{1}(h)\right)$ and $\pi_{<\delta}^{1}(H)=\pi_{<\delta}\left(\pi^{1}(H)\right) ; F^{\prime}=\pi_{<\delta}^{1}\left(F_{\geq \delta}\right)$ and $G^{\prime}=\pi_{<\delta}^{1}\left(G_{\geq \delta}\right)$. Then $\pi_{<\delta}^{1}(a) \in \overline{F^{\prime}} \cap \overline{G^{\prime}}$. Both sets $F_{\geq \delta}$ and $G_{\geq \delta}$ are closed in $X$, therefore they are countably compact. Then $F^{\prime}, G^{\prime}$ are countably compact too. This implies that $F^{\prime}, G^{\prime}$ are closed in $\pi_{<\delta}^{1}\left(X_{\geq \delta}\right)=\Sigma\left\{D_{\alpha}: \alpha \in \delta\right\} \ni 1^{\delta}$ since the latter is Frechet. $\pi_{<\delta}^{1}(X)$ is also normal and $C$-embedded in $\prod\left\{D_{\alpha}: \alpha \in \delta\right\}$; hence $F^{\prime} \cap G^{\prime} \neq \emptyset$. Therefore there are $x \in F_{\geq \delta}$ and $y \in G_{\geq \delta}$ such that $\pi_{<\delta}^{1}(x)=\pi_{<\delta}^{1}(y)$. So we can choose sequences $\left\{x_{n}: n \in \omega\right\} \subset F$ and $\left\{y_{n}: n \in \omega\right\} \subset G$ and an index sequence $\left\{\delta_{n}: n \in \omega\right\}$ such that $\pi_{<\delta_{n}}^{1}\left(x_{n}\right)=\pi_{<\delta_{n}}^{1}\left(y_{n}\right)$ for all $n \in \omega$, such that $\left\{\pi^{2}\left(x_{n}\right): n \in \omega\right\},\left\{\pi^{2}\left(y_{n}\right): n \in \omega\right\}$ and $\left\{\delta_{n}: n \in \omega\right\}$ are nondecreasing and such that $\sup \left(\left\{\pi_{2}\left(x_{n}\right): n \in \omega\right\}\right)=\sup \left(\left\{\pi_{2}\left(y_{n}\right): n \in \omega\right\}\right)=\sup \left(\left\{\delta_{n}: n \in \omega\right\}\right)=\gamma^{\prime}$ for some $\gamma^{\prime} \in \omega_{2}^{\prime}$. Similar to the first part of this Claim, the sequences $\left\{x_{n}: n \in \omega\right\}$ and $\left\{y_{n}: n \in \omega\right\}$ contain subsequences which converge to some point $b \in X_{\gamma^{\prime}}$. Then $b \in F \cap G$ since $X$ is countably compact. This contradiction proves that $\bar{F}^{K} \cap \bar{G}^{K}=\emptyset$, and therefore $X$ is normal $C$-embedded in $K$.

It follows from Claim 1 that $T$ is countably compact.

Claim 2. $T$ is normal.

Proof. Assume the contrary. Then there are closed disjoint $F, G \subset T$ such that $\bar{F}^{K} \cap \bar{G}^{K} \neq \emptyset$. Let $a \in \bar{F}^{K} \cap \bar{G}^{K}$. Since both $X$ and $Y$ are normal and $C$ embedded in $K$, we have that $\overline{F \cap X}^{K} \cap \overline{G \cap X}^{K}=\emptyset$ and $\overline{F \cap Y}^{K} \cap \overline{G \cap Y}^{K}=\emptyset$. Then there is a closed neighborhood $H$ of $a$ in $K$ which is disjoint from one of the sets $\overline{F \cap X}^{K}$ or $\overline{G \cap X}^{K}$ and from one of the sets $\overline{F \cap Y}^{K}$ or $\overline{G \cap Y}^{K}$. Then the sets $F \cap H$ and $G \cap H$ still contain $a$ in their closures and one of them is contained in $X$, the other in $Y$. So we can assume from the beginning that $F \subset X$ and that $G \subset Y$. For each $\delta \in \omega_{2}$ let $F^{\delta}=\left\{x \in X: \pi^{2}(x) \geq \delta\right.$ and $\left.\pi_{<\delta}\left(\pi^{1}(x)\right) \in \pi_{<\delta}\left(Y^{\prime}\right)\right\}$. Then $F^{\delta}$ is closed in $X$ and $Y \subset{\overline{F^{\delta}}}^{K}$. Since $X$ is normal and $C$-embedded in $K$, this implies that $F \cap F^{\delta} \neq \emptyset$. So for each $\delta \in \omega_{2}$ we can pick $x_{\delta} \in F \cap F^{\delta}$ and form the set $F^{\prime}=\left\{x_{\delta}: \delta \in \omega_{2}\right\}$. Consider $F^{\prime \prime}=\left\{x_{\delta}^{\prime \prime} \in K^{\prime}: \delta<\omega_{2}\right\}$, where $x_{\delta}^{\prime \prime}=\left(\pi_{<\delta}\left(\pi^{1}\left(x_{\delta}\right)\right), 1^{\omega_{2} \backslash \delta}\right)$ (where $\left.x_{\delta} \in F^{\prime}\right)$. Clearly, $F^{\prime \prime} \subset X^{\prime} \cap Y^{\prime}$. If there is $b \in Y^{\prime}$ such that $\left|\left\{\delta \in \omega_{2}: x_{\delta}^{\prime \prime}=b\right\}\right|=\omega_{2}$, then it is easy to see that each neighborhood of $\left(b, \omega_{2}\right)$ intersects $F^{\prime}$, so $\left(b, \omega_{2}\right) \in{\overline{F^{\prime}}}^{K} \cap Y$. Otherwise, $\left|F^{\prime \prime}\right|=\omega_{2}$ and there is a complete accumulation point $c$ of $F^{\prime \prime}$ in $X^{\prime} \cap Y^{\prime}$ by Chertanov's 
theorem (which states that the Lindelöf number of a $\Sigma$-product of any number of compacta is not greater than $\omega_{1}$ ) and since $X^{\prime} \cap Y^{\prime}$ is a closed subset of $X^{\prime}$. Then $\left(c, \omega_{2}\right) \in{\overline{F^{\prime}}}^{K} \cap Y$. In both cases ${\overline{F^{\prime}}}^{T} \cap Y \neq \emptyset$, which contradicts the assumption that $F \subset X$. Therefore $T$ is normal.

Now we show that $T$ does not possess property $(a)$. Since $X$ is dense in $K$, it is sufficient to produce an open cover $\mathcal{U}$ of $T$ such that for any finite $Z \subset X, S t(Z, \mathcal{U}) \not \supset$ $T$. For $\alpha \in \omega_{2}^{1}$ and $\beta \in \omega_{2}^{2}$, let $U_{\alpha}=\left(\pi^{1}\right)^{-1}\left(\pi_{\alpha}^{-1}(0)\right)$ and $U_{\beta}=\left(\pi^{1}\right)^{-1}\left(\pi_{\beta}^{-1}(1)\right)$. Let $\mathcal{U}_{1}=\left\{T \cap U_{\alpha}: \alpha \in \omega_{2}^{1}\right\}, \mathcal{U}_{2}=\left\{T \cap U_{\beta}: \beta \in \omega_{2}^{2}\right\}$. Then $\mathcal{U}=\mathcal{U}_{1} \cup \mathcal{U}_{2}$ is an open cover of $T$. It is easy to see that $Y \cap\left(\cup \mathcal{U}_{1}\right)=\emptyset$. For finite $Z \subset X$ let $\beta=\max \left(\pi^{2}(Z)\right)$. Then $|\beta| \leq \omega_{1}$; therefore the point $\left(1^{\omega_{2}^{1} \cap \beta} \times 0^{\omega_{2}^{2} \cap \beta} \times 1^{\omega_{2} \backslash \beta}, \omega_{2}\right)$ is in $Y \backslash S t(Z, \mathcal{U})$ and $T$ does not have property $(a)$.

Claims 1 and 2 yield the following:

Theorem 1. There is a normal countably compact not absolutely countably compact space.

\section{Further RESUlts}

Theorem 2. Every hereditarily normal countably compact space is absolutely countably compact.

Proof. Let $X$ be a hereditarily normal countably compact space. Let $F$ be a dense subset of $X$ and let $\mathcal{U}$ be an open cover of $X$. It was noted in [RSV] that there is a finite $G \subset F$ such that $S t(G, \mathcal{U})$ contains $[F]_{\aleph_{0}}$, where $[F]_{\aleph_{0}}$ denotes $\cup\left\{\bar{H}^{X}: H \subset\right.$ $F, H$ is countable $\}$. Assume that $X \not \subset S t(P, \mathcal{U})$ for any finite $P \subset F$; then the set $H=X \backslash S t(G, \mathcal{U})$ is infinite. Therefore we can choose a family $\mathcal{V}=\left\{V_{n}: n \in \omega\right\}$ of open subsets of $X$ such that $\left\{\overline{V_{n}}: n \in \omega\right\}$ is discrete in its union and such that $H \cap V_{n} \neq \emptyset$ for all $n \in \omega$. Let $D=\overline{\cup \mathcal{V}} \backslash\left(\cup\left\{\overline{V_{n}}: n \in \omega\right\}\right)$. For each $n \in \omega$, let $a_{n} \in H \cap V_{n}$. The sets $A=\left\{a_{n}: n \in \omega\right\}$ and $B=(X \backslash \cup \mathcal{V}) \backslash(H \cap D)$ are disjoint and closed in $X \backslash(H \cap D)$. Therefore $A$ and $B$ are contained in disjoint neighborhoods $W_{1}, W_{2}$ in $X \backslash(H \cap D)$. For each $n \in \omega$ we pick $c_{n} \in V_{n} \cap W_{1} \cap F$. The set $C=\left\{c_{n}: n \in \omega\right\}$ is discrete and closed in $X \backslash(H \cap D)$. This is a contradiction since $C \subset F$ and since $\bar{C}^{X} \subset[F]_{\aleph_{0}} \subset X \backslash(H \cap D)$.

Theorem 3. Let $\tau$ be an infinite cardinal, $l(X) \geq \tau$, and assume that $X$ contains a dense subset $G$ which satisfies one of the following conditions:

(1) $|G|<\tau$.

(2) $|G|=\tau$ and for each $H \subset G,|H|=\tau \Rightarrow H$ has a complete accumulation point in $X$.

Then there is a space $M \supset X$ without property (a) such that all points of $M \backslash X$ are isolated. Also if $X$ is (hereditarily) normal, then $M$ is (hereditarily) normal too.

Proof. According to our assumptions, there is a cardinal $\mu \geq \tau$ and an open cover $\mathcal{U}=\left\{U_{\alpha}: \alpha<\mu\right\}$ without a subcover of smaller cardinality. Let $A$ be a space of cardinality $\mu$ with a single nonisolated point $a$ such that $U$ is a neighborhood of $a$ in $A$ iff $a \in U$ and $|A \backslash U|<\mu$. We denote $M^{\prime}=X \times A$ with the product topology plus all points of $X \times(A \backslash\{a\})$ are isolated. Further, $M \subset M^{\prime}$, $M=(X \times\{a\}) \cup(G \times A)$. We claim that $M$ has the required properties. Let $\pi^{1}$, $\pi^{2}$ denote projections of $M^{\prime}$ on $X$ and $A$ respectively. Let $A \backslash\{a\}$ be enumerated: 
$A \backslash\{a\}=\left\{a_{\alpha}: \alpha<\mu\right\}$. For each $\alpha<\mu$ let $U_{\alpha}^{\prime}=\left(U_{\alpha} \times\left(A \backslash\left\{a_{\beta}: \beta<\alpha\right\}\right)\right) \cap M$. Let $\mathcal{U}^{\prime}=\left\{U_{\alpha}^{\prime}: \alpha<\mu\right\}$ and $\mathcal{U}^{\prime \prime}=\left\{\{y\}: y \in M \backslash \cup \mathcal{U}^{\prime}\right\}$. Clearly $\mathcal{U}^{\prime} \cup \mathcal{U}^{\prime \prime}$ is an open cover of $M$. For any $a^{\prime} \in(A \backslash\{a\})$ the set $\left\{U \in \mathcal{U}^{\prime}: a^{\prime} \in \pi^{2}(U)\right\}$ is of cardinality less than $\mu$. Therefore if $F \subset G \times(A \backslash\{a\})$ and $\left|\pi^{2}(F)\right|<\mu$, then $\operatorname{St}\left(F, \mathcal{U}^{\prime} \cup \mathcal{U}^{\prime \prime}\right) \not \supset X \times\{a\}$. However, if $F \subset G \times(A \backslash\{a\})$ and $\left|\pi^{2}(F)\right|=\mu$, then $F$ has an accumulation point in $X \times\{a\}$ from (i) and (ii). This proves that $M$ does not have property $(a)$. Now assume that $X$ is normal. If $G_{1}, G_{2}$ are disjoint closed subsets of $M$, then $G_{1} \cap(X \times\{a\})$ and $G_{2} \cap(X \times\{a\})$ are disjoint and closed in $X \times\{a\}$ (one or both of these sets may be empty). Let $U^{1}, U^{2}$ be disjoint open neighborhoods of $G_{1} \cap(X \times\{a\}), G_{2} \cap(X \times\{a\})$ in $X \times\{a\}$. Then sets

$$
\left\{\left[\left(U^{1} \times A\right) \backslash G_{2}\right] \cup\left[G_{1} \cap(G \times(A \backslash\{a\}))\right]\right\} \cap M
$$

and

$$
\left\{\left[\left(U^{2} \times A\right) \backslash G_{1}\right] \cup\left[G_{2} \cap(G \times(A \backslash\{a\}))\right]\right\} \cap M
$$

are disjoint open neighborhoods of $G_{1}, G_{2}$ in $M$. Therefore $M$ is normal. A similar argument shows that $M$ is hereditarily normal when so is $X$. The theorem is proved.

Clearly, any hereditarily normal space $X$ such that $d(X)<l(X)$ satisfies the conditions of Theorem 3. Examples of such $X$ are abundant under various additional set-theoretic assumptions. It would be of great interest, however, to have such an example in ZFC.

Question 2. Is there a ZFC example of a hereditarily normal space $X$ such that $d(X)<l(X)$ ?

The author is grateful to P. Szeptycki for interesting and motivating discussions and for help and to J. Vaughan for useful suggestions.

\section{REFERENCES}

[Eng] Ryszard Engelking, General Topology, Heldermann Verlag, Berlin, 1989. MR 91c:54001

[Fle] Fleischman W. M., A new extension of countable compactness, Fund. Math. 67 (1970), 1-9. MR 41:9200

[JMS] Winfried Just, Michael V. Matveev, and Paul J. Szeptycki, Some results on property (a), preprint.

[Mat1] Michael V. Matveev, Absolutely countably compact spaces, Topology Appl. 58 (1994), 81-92. MR 95e:54035

[Mat2] Michael M. Matveev, Some questions on property (a), Questions Answers Gen. Topology 15 (1997), 103-111. MR 98k:54035

[Pac] W. Pack, Absolutely countably compact topological groups, preprint.

[RSV] Mary Ellen Rudin, Ian S. Stares and Jerry E. Vaughan, From countable compactness to absolute countable compactness, Proc. Amer. Math. Soc 125 (1997), 927-934. MR 98c:54017

[Vau] Jerry E. Vaughan, On the product of a compact space with an absolutely countably compact space, Proceedings of the Papers on General Topology and Applications, New York Academy of Sciences $\mathbf{7 8 8}$ (1996), 203-208. MR 98g:54046

Department of Mathematics, Ohio University, Morton Hall, Athens, Ohio 45701-2979

E-mail address: opavlov@bing.math.ohiou.edu 